\title{
Prevention of extra-analytical phase errors by non-analytical automation in clinical laboratory
}

\section{Klinik laboratuvarda non-analitik otomasyon ile extra-analitik faz hatalarının önlenmesi}

https://doi.org/10.1515/tjb-2020-0483

Received March 20, 2020; accepted December 26, 2020;

published online January 20, 2021

\begin{abstract}
During previous decades, significant improvements in laboratory errors have become a substantial part of reducing preventable diagnostic errors. In clinical laboratory practice, the errors in the testing process are primarily associated with extra-analytical phase error sources, influencing the test result quality profoundly. Thus, the management of these critical error sources makes their effects preventable thanks to automation and computer sciences. The implementation of non-analytical automated systems requires a risk management strategy based on laboratory's workflow and bottlenecks. Then, the improvements can be measured and evaluated by the usage of quality indicators (QI). Consequently, the total quality of laboratory diagnostics and higher patient safety is closely dependent on this type of automation. This review will help laboratory professionals, managers, and directors improve the total testing processes (TTP). The automation technologies have added a serious impact on the proficiency of laboratory medicine. Several instrumentations have now partially or entirely automated many manual tasks to improve standardization, organization, efficiency, and TTP quality. The implementation of non-analytical automation has made them manageable.
\end{abstract}

\footnotetext{
*Corresponding author: Ebubekir Bakan, Faculty of Medicine, Department of Medical Biochemistry, Atatürk Üniversitesi, Erzurum, Turkey, E-mail: ebubekirbakan@gmail.com. https://orcid.org/00000002-6390-9954

Nuri Bakan, Faculty of Medicine, Department of Medical Biochemistry, Atatürk Üniversitesi, Erzurum, Turkey
}

As a result, non-analytical automation within and outside the clinical laboratory will necessarily lessen the error sources' effect on the total test process, enhancing the quality of the test results.

Keywords: model quality indicators; non-analytical automation; pre-analytical error sources; quality indicators; total testing process.

\section{Özet}

Önceki on yıllarda, laboratuvar hatalarındaki önemli gelişmeler, önlenebilir teşhis hatalarını azaltmanın önemli bir parçası haline gelmiştir. Klinik laboratuvar uygulamasinda, test sürecindeki hatalar öncelikle ekstra analitik faz hata kaynakları ile ilişkilendirilir ve test sonuç kalitesini derinden etkiler. Böylelikle bu kritik hata kaynaklarının yönetimi, otomasyon ve bilgisayar bilimleri sayesinde etkilerini önlenebilir hale getirir. Non-analitik otomatik sistemlerin uygulanması, laboratuvarın iş akışına ve darboğazlara dayalı bir risk yönetimi stratejisi gerektirir. Ardından kalite göstergeleri kullanılarak iyileştirmeler ölçülebilir ve değerlendirilebilir. Sonuç olarak, laboratuvar teşhislerinin toplam kalitesi ve daha yüksek hasta güvenliği, bu tip otomasyonla yakından bağlıdır. Bu derleme makalesi, laboratuvar uzmanlarının, yöneticilerinin ve direktörlerinin toplam test süreçlerini iyileştirmesine yardımcı olacaktır. Otomasyon teknolojileri, laboratuar tıbbının yeterliliği üzerinde ciddi bir etki yaratmıștır. Pek çok enstrümantasyon, toplam test süreci standardizasyonu, organizasyonu, verimliliği ve kalitesini iyileştirmek için birçok manuel iş ve işlemi kısmen veya tamamen otomatikleştirmiştir. Non-analitik otomasyonun uygulanması bu işlemleri yönetilebilir hale getirmiştir. Sonuç olarak, klinik laboratuvar içindeki ve dışındaki nonanalitik otomasyon, hata kaynaklarının toplam test süreci 
üzerindeki etkisini kaçınılmaz olarak azaltacak ve test sonuçlarının kalitesini artıracaktır.

Anahtar kelimeler: kalite indikatörleri; model kalite indikatörleri; nonanalitik otomasyon; preanalitik hata kaynakları; toplam test süreci.

\section{Introduction}

This review article aims to introduce the importance of laboratory error prevention based on non-analytical automation. First, the error sources/errors in the extra-analytical phase of the clinical laboratory will be detailed. Secondly, some possible improvements in these errors by non-analytical automation will be focused on. Finally, the conclusion will be included. Table 1 shows some definitions of clinical laboratory automation and the issue, which the reader can consult for clearance.

Automation comprises extra-analytical and analytical processes, with the latter being developed earlier than the former [1]. Clinical laboratory automation has improved such laboratory processes as specimen labeling, sorting, transport, processing, loading on the analyzers, analysis, storage, and archiving [2]. Although the analytical and non-analytical automation has developed asynchronously, clinical laboratory performances have dramatically changed to a satisfactory level today [3].

Previously, the term automation was used for clinical chemistry analyzers to describe the processes with only minimal involvement of an analyst. The automation, for the past two decades, has also covered extra-analytical as well as analytical procedures. It is well known that extra-analytical error sources dominate those of analytical systems and have become substantial for the efficiency of clinical laboratories $[1,4,5]$. Since a high-quality test result can be defined as the fact that it must be accurate and be reported faster, i.e., short turnaround time (TAT), the value of extra-analytical technologies besides the automated analyzers may be appreciated well. Consequently, the implementation of automatable processes to the extra-analytical phase will become crucial, considering the improvement of clinical laboratory performance and patient's sample safety [6].

Extra-analytical and analytical components have recently been integrated or interfaced with each other to construct fully integrated modular laboratory automation. The current developments in non-analytical automation provide error elimination, improve the quality, and reduce the labor, costs, and TAT [3]. On the one hand, a wide range of variables is included in the extra-analytical phase, such as test order and sample collection, delivery to the
Table 1: Some definitions associated with clinical laboratory automation.

Term
Laboratory automation
Total laboratory automa-
tion (TLA)

\section{Definition}

The general term used for automated clinical laboratory (instrumentation + LIS/HIS)

Laboratory automation composed of heterogeneous, physically-integrated analytical and extra-analytical systems (many analyzers performing different types of tests on different sample matrices)

Analytical automation

Automated analytical systems or workstations

Non-analytical

automation

Part of laboratory automation other than analyzers; signifies pre- and post-analytical automation

Intra-laboratory nonanalytical automation Pre-analytical automation within the clinical laboratory (pre- and post-analytical automation)

Extra-laboratory nonanalytical automation

Pre-analytical automation outside the clinical laboratory; signifies prepreanalytical phase automation

Extra-analytical phase Intra- and extra-laboratory processes other than analytic processes

On-line analyzer

An analyzer interfacing the integrated laboratory automation system

Off-line analyzer

An analyzer interfacing with no integrated laboratory automation system; stand-alone analyzer

Integrated/modular laboratory automation

An automation composed of

pre-analytical, analytical and post-analytical component linked by a conveyor

Modular analytical system

An analyzer designed as modules; module addition or exclusion possibility depending on the need

Modular non-analytical system

A non-analytical system designed as modules; module addition or omission possibility depending on the need

Stand-alone preanalytical system A pre-analytical specimen processor interfacing no analyzer

The duration in which the laboratory reports the test result

Analytical TAT Testing duration beginning with sample load to the analyzer and ending with readout.

Intra-laboratory TAT Reporting duration beginning with sample submission by laboratory and ending with report verification.

Total TAT Reporting duration beginning with either test ordering or specimen collection and ending with report verification.

TLA, total laboratory automation; TAT, Turnaround time.

laboratory, handling, and processing before analysis. On the other hand, the pre-analytical part of the extraanalytical-phase covers all processes outside and inside the 
clinical laboratory or begins with test order and ends with analyzer entrance of the processed samples. Similarly, the post-analytical part of the extra-analytical phase comprises auto-verification, recapping, automated specimens archiving and storage, decapping in the case of a rerun, and secondary specimen sorting for off-line analyzers $[1,3,7]$.

In short, extra-analytical processes summarized in Table 2 can be automated by non-analytical instrumentation in the clinical laboratory together with some limitations depending on the facilities. Either of the following automation can automate some of these processes: (a) stand-alone pre-analytical systems (single-function or multifunction) [24-28] and (b) integrated pre- and post-analytical systems $[1,3,27,29-32]$. Consequently, we think that many extraanalytical processes, within and outside the clinical laboratory, have nowadays been or may in the future, be automated. Resultantly, the extra-analytical phase management may prevent some errors, resulting in the total quality of laboratory diagnostics and high-quality test results, as considered in the following sections. For this reason, the review has been intended especially for laboratory professionals, managers, and directors for the sake of patient safety and will help improve the TTP in the laboratory.

(1) Error sources/errors in the extra-analytical phase of clinical laboratory.

In the clinical laboratory, each step in total testing processes (TTP) may be affected by laboratory errors. However, a large body of evidence has shown that the extra-analytical phase (both intra- and extra-laboratory) are more prone to errors than the analytical phase, as mentioned earlier [7, 25-28, 29-31]. Fortunately, those kinds of errors have been able to be prevented partly by the introduction of some technological and computer-aided informatic solutions to that phase. Several studies have shown that after the

Table 2: Extra-analytical, automatable processes associated with clinical laboratory.

\begin{tabular}{lr}
\hline Extra-analytical, automatable processes & References \\
\hline Specimen sampling & {$[8,9]$} \\
Specimen identification & {$[10-13]$} \\
Tube labeler and preparer & {$[14,15]$} \\
Specimen delivery & {$[16-19]$} \\
Tube loader and sorter & {$[20]$} \\
Use of whole blood for analysis & {$[21]$} \\
Process-controlling software in integrated/modular & {$[22,23]$} \\
laboratory automation systems & \\
Non-analytical automation in integrated/modular & \\
laboratory automation systems & \\
Specimen preparation & \\
Automated specimen storage and retrieval & \\
\hline
\end{tabular}

implementation of stand-alone pre-analytical systems to clinical laboratories, the errors in the conventional pre-analytical steps have been considerably reduced [32-34].

Similarly, some of the technological and computer-aided bioinformatic solutions have been able to be implemented to the extra-laboratory part of the non-analytical phase, resulting in partial automation in that phase. On the other hand, the extra-laboratory part of the extra-analytical phase is not under the control of laboratory staff since other nonlaboratory health care stuff execute the processes at this phase, covering several processes, including test order and specimen collection, identification, labeling, handling, tracing, and transporting. Consequently, laboratory professionals and staff cannot directly control and are not responsible for that phase's errors. However, it is of critical importance for the laboratory professionals to partly standardize and control the extra-laboratory steps [29, 34-36]. By using bioinformatic technology and robotics, thousands of clinical laboratories worldwide could have the opportunity to reduce errors caused by pre-analytical processes, resulting in improvement in accuracy and TTP. Unfortunately, there are limited numbers of studies focusing on the error improvements by implemented tools. Before the implementation of such automation, each laboratory does need justification for demonstrable improvements. However, it is clear that non-analytical automation effectively reduces the number of laboratory errors occurring during TTP [31, 32].

Lippi et al. [7] summarized major sources of pre-analytical variability, associated with patient preparation, sample collection, sample transportation, sample preparation for analysis, and sample storage. Under these main categories, Lippi et al. detailed 20 different sub-variables. The clinicians and the patients are generally interested in total testing error and timeliness (i.e., TAT), which are the basis of test quality or the measure of performance of the clinical laboratory. Automation of analytical and extra-analytical phases of laboratory processes enhanced clinician and patient satisfaction, which is because prompt, electronic reporting of test results and critical value alerting by bioinformatics as well as test quality are of critical importance $[28,37]$. Thus, TAT improvement may be considered as a quality indicator (QI), a measure of laboratory performance. The implementation of performance measurements must evaluate TTP.

For this reason, the guidelines and regulations on the clinical laboratory test performance encourage the laboratories to improve all steps of the TTP as well as the analytical phase [38]. International Standard for Accreditation of Clinical Laboratories (ISO 15189:2012) prompts the laboratorians to establish QIs to assess clinical laboratory performance and to reduce the errors caused by the extra-analytical phase. 
The standard similarly states that the laboratory shall establish QIs to monitor and evaluate performance throughout critical aspects of pre-examination, examination, and post-examination processes. It also suggests the process of monitoring QIs be planned, which includes establishing the objectives, methodology, interpretation, limits, action plan, and duration of measurement [39-43].

In the application of QIs to all clinical laboratories thoroughly, one can have difficulties or discrepancies since a laboratory's QIs assessment belongs to its establishment and not applicable to other laboratories. Resultantly, a harmonization of the usage of IQs is needed. For this purpose, the Working Group on Laboratory Errors and Patient Safety (WG-LEPS) of the International Federation of Clinical Chemistry and Laboratory Medicine (IFCC) planned a project in 2008 for establishing the Model of QI (MQI) $[44,45]$. The project was targeted to define a list of MQIs and to determine the data collection procedures and quality specifications for laboratory result evaluation. In succeeding years, the MQIs were discussed and approved in the 2013 Padova Consensus Conference and were tested on different occasions. Thus, the resultant MQI covers 53 QIs, which are generally key processes. Of them, 39 are related to the extra-analytical (pre- and post-analytical) phase, which is of critical importance. The MQIs have been listed by classification based on TTP phases. Besides, the significance of a given QI and data collection difficulty determine its order of priority, grading from 1 to 4 (one the highest, four the lowest priority). The QIs, for example, with priority 1 is mandatory and the first to be put into practice [46]. MQIs of critical processes associated with the pre-analytical phase include misidentification errors, test transcription errors, incorrect sample type, incorrect fill level, unsuitable samples for transportation and storage problems, sample hemolysed, and inappropriate time in sample collection, and almost all of which have priority 1 . On the other hand, MQIs of critical processes associated with post-analytical phase processes encompass inappropriate TATs, incorrect laboratory reports, notification of critical values, and results notification (TAT), with the majority having priority 1 as well.

After the implementation of automation to the extraanalytical phase, the MQI ratios will become low when compared with those of non-automated processes, since MQIs have rather good match with extra-analytical phase errors. A study describing the comparison of the pre- and post-automation QI evaluation of the same clinical laboratory would be valuable and show the importance of non-analytical automation.

(2) Improvements in extra-analytical phase errors by non-analytical automation.
The non-analytical automation, within and outside the clinical laboratory, will substantially lessen the effect of the error sources in TTP and enhance the quality of the test results, resulting in an accurate result(s) and faster test result reporting. For the implementation of an MQI, a QI form is prepared first, which includes many characteristics such as identification code, method of data collection, a method for data processing, the goal for improvement activities, and so on. The classification of the QI in that form is also marked as Efficiency, Structure, Effectiveness, Activity/process, Timeliness (TAT), Results, Pre-analytical phase, Intra-analytical phase, Post-analytical phase, Safety of the staff, Outcome, Support processes, and competence. There may be a relationship between some of these classification items (e.g., Timeliness, Preanalytical phase, Post-analytical phase, and Safety of the staff) and the improvements executed by non-analytical automation tools. On the other hand, there is a good match between pre-analytical error types and their amelioration by non-analytical automation. Similarly, there is a considerable overlap of pre-analytical error types with MQIs. The majority of the non-analytical automation steps are closely associated with increased patient and healthcare staff safety.

Lippi, G. et al. reported the pre-analytical error types [41]. We divided these error types into two categories: intraand extra-laboratory extra-analytical errors. Two tables were instructed (Tables 3 and 4), describing the errors and their prevention. The extra-laboratory errors occur in the pre-pre-analytical phase, and intra-laboratory errors in the intra-aboratory pre- and post-analytical phases. Each error type mentioned in Tables 3 and 4 were illuminated by a detailed comment or explanation about the probable improvements provided by non-analytical automation at the respective steps for lessening the error ratio. We can summarize this situation as follows.

Ten of 12 extra-laboratory extra-analytical errors in Table 3 and all intra-laboratory non-analytical errors in Table 4 may be prevented by automation. İmprovements can be summarized as follows, which are detailed in two tables. Missing sample and/or test request is prevented by computerized physician order entry and query-host communication. Positive patient identification by bioinformatics prevents wrong/missing identification of the patients. In vitro hemolysis may partly be averted by adaptor usage, using automated venous sampling systems, and using devices to find the veins or real-time digital imagers for sampling. All are closely associated with both patient and healthcare staff safety and high-quality testing. Similarly, the tube labeler and preparer tool hampers the wrong container issue, related to correct patient ID and tube labeling for phlebotomy and meaning 
Table 3: Extra-laboratory extra-analytical errors and their prevention by automation.

\section{Extra-laboratory error types or Comments and explanation of the inconveniences improvement manner}

1. Missing sample and/or test request

Computerized physician order entry and query-host communication may help reduce this type of error.

2. Wrong/missing identification Positive patient identification provided by several technologies may prevent this type of error.

3. In vitro hemolysis

4. Clotting in anticoagulated sample

5. Wrong container

6. Contamination from infusion route

7. Insufficient sample
There are several causes of them may be sampling with the injector and pouring the specimen into the vacutainer without removing the needle. If an adaptor is used for sampling, this type of error may partly be prevented, and different kinds of specimens can be taken in the same setting from the same patient. That adaptor may be considered as a local, automating toll. Additionally, if the safety needle is used for sampling together with an adaptor, the stuff's safety will be provided. Automated venous sampling systems and devices to find the veins, or real-time digital imagers, for sampling are closely associated with both patient and healthcare staff safety and prevent hemolysis, adding an impact value to TTP and providing high-quality testing. Dependent on inefficient mixing immediately after sampling. No automation is possible for this error.

The tube labeler and preparer, an automated, LIS/HIS-connected system, may reduce this error ratio. The system is widely used for outpatients. Patient safety in TTP requires correct patient ID and tube labeling for phlebotomy.

Dependent on the staff of clinics during sampling from inpatients. No automation is possible for this error.

The vaccum within the container determines the sipping volume of the vacutainer. Adaptor usage for sampling may prevent insufficient sample. The phlebotomist waits for complete filling. The higher vacuum volumes are necessary in vacutainers in high-altitude regions than those at sea-level regions. For hemolysis during sampling. One of

Table 3: (continued)

\section{Extra-laboratory error types or Comments and explanation of the inconveniences improvement manner}

instance, while a $2 \mathrm{~mL}$-vacuum is enough for complete blood cell counting tubes at sea level, at least a $3 \mathrm{~mL}$-vacuum volume is necessary for that kind of sampling at 2,000 m altitude (by our experience). It is possible to make the manufacturer manufacture high volume-vacuum tubes when ordered.

8. Inappropriate blood to anticoagulant ratio

9. Insufficient mixing of the sample

10. Inappropriate transport and It is associated with transport storage conditions

12. The low-resolution print quality of the bar-code label

The same solution as that of insufficient sample error may be suggested for improper blood to anticoagulant ratio. The phlebotomist must use an adaptor for sampling and wait for complete filling of the tube vacuum. No automation is possible for this error. The mixing is a staffdependent situation. systems for specimen delivery to the laboratory. Pneumatic tube system (PTS) (or conveyors) and robots, which are automated specimen transport systems, can get rid of it. Transportation monitoring systems can help trace the samples in this traveling. The PTSs are fast transporter all laboratory specimens (outpatients and inpatients). For safe transportation by PTS, a carrier with a high radius (about $160 \mathrm{~mm}$ ), soft material support within the carrier, low or optimized acceleration and deceleration, non-sharpened corners or bending points in the tubing network may be critical.

11. Inadequate label alignment The tube labeler and preparer may on the specimen container lessen the errors associated with inadequate label alignment on specimen containers, especially for outpatients. Inappropriate label alignment means no reading by the bar-code readers located in the total laboratory automation system. Removal of this error source by automated devices shortens total TAT.

The tube labeler and preparer may lessen the errors associated with the bar-code label's low-resolution print quality, especially for outpatients. Inappropriate label print means no reading by the bar-code readers located in the total 
Table 3: (continued)

\begin{tabular}{ll}
\hline $\begin{array}{l}\text { Extra-laboratory error types or } \\
\text { inconveniences }\end{array}$ & $\begin{array}{l}\text { Comments and explanation of the } \\
\text { improvement manner }\end{array}$ \\
\hline & $\begin{array}{l}\text { laboratory automation system. } \\
\text { Removal of this error source by } \\
\text { automated devices improves total } \\
\text { TAT. }\end{array}$
\end{tabular}

TTP, total testing processes; PTS, pneumatic tube system; TAT, Turnaround time.

Table 4: Intra-laboratory extra-analytical errors and their prevention by automation.

\begin{tabular}{ll}
\hline $\begin{array}{l}\text { Intra-laboratory error types or } \\
\text { inconveniences }\end{array}$ & $\begin{array}{l}\text { Comments and explanation of the } \\
\text { improvement manner }\end{array}$ \\
\hline
\end{tabular}

1. Inappropriate centrifugation In automated centrifugation, the conditions

2. Inappropriate storage condition in the posttest phase

3. Delay in sample processing steps in laboratory

4. Delayed submission and inefficient specimen traceability

Table 4: (continued)

\section{Intra-laboratory error types or Comments and explanation of the} inconveniences

improvement manner

5. Inability to process primary Similar to analytical systems, tube non-analytical automation devices can also process primary tubes, which eliminates aliquoting steps, and the on-line analyzers can directly use these tubes for sampling, which shortens TAT. These tubes can also be directly stored in the storage cabinet.

6. Errors from inadequate sample integrity standardization of the requirements such as balancing is possible, which is necessary for specimen (naturally patient) safety. This system prevents broken samples. Generally, the capacity of automated system centrifuges is remarkably high, providing shortened TAT and staff safety.

It is associated with automated specimen storage and retrieval, which makes it easy that the storage conditions are standardized, and that the retrieval is fastened. The robotics of the system finds a specimen within seconds for a rerun, dilution, add-on tests, etc. Appropriate storage conditions are obtained, such as dark storage, refrigeration, closed samples, meaning sample safety, and improved TAT.

The tube loader and sorter can submit the specimen containers and their sorting for laboratory devices based on defined criteria. It is a very fast (throughput higher than 1,500 samples per hour) and an efficient tool, improving the workflow in clinical laboratories, particularly in the pre-analytical phase. It is a tasktargeted modular system. The inappropriate specimens are rejected and are not submitted to the laboratory. Thus, the sample sorting and accepting of the laboratory is critical and must be accelerated to make TAT reduce and achieve staff safety.

The tube loader and sorter execute the submission function and provide efficient specimen traceability. processes sample traceability

9. Delayed test reporting laboratory automation

TAT, Turnaround time.
This module of the pre-analytical system checks sample integrity. Specimen quality is critical for producing a high-quality test result. Unnecessary repetitions of tests are prevented by this module, improving the test results and TAT.

7. Errors from different manual Different mechanical tasks are

carried out using this module, and all processes involved are accelerated, reducing TAT. Decapper, aliquoter, bar-code labeler, recapper units of modular pre-analytical prevent these errors.

8. Impairment in secondary Secondary tubes generated by the aliquoting unit are sorted to off-line analyzers's racks. Possible chaos and sample loss for rare tests are prevented. Specimen safety is provided, and resampling is prevented.

Auto-verification is essential for clinical laboratory automation and is based on computer sciences, with no manual intervention. The auto-verification either sends the patients' reports directly to the clinician's desktop or blocks them for the laboratorian to concentrate on the pathological results. In direct reporting, the results bear defined criteria for acceptance of the test results as non-pathological, meaning greatly decreased inspection time for test results or improved TAT.

10. The deceleration in total

Process-managing software connects the LIS to the fullyautomated system composed of pre-analytical, analytical, and post-analytical components. It considers the whole system as if it were a single device. A single operator can manage the entire system. It is a kind of integrator, providing the integration of TTP with maximal effectiveness. All factors improved result in high-quality test reporting. 
Table 5: Summary of extra-analytical errors, improvements by automation, and improved parameters.

\begin{tabular}{|c|c|c|c|c|c|}
\hline \multirow{2}{*}{$\begin{array}{l}\text { Extra-laboratory error types or } \\
\text { inconveniences }\end{array}$} & \multirow[t]{2}{*}{ Improvement by automation } & \multicolumn{4}{|c|}{ Improvements in } \\
\hline & & TAT & $\begin{array}{l}\text { Test result } \\
\text { quality }\end{array}$ & $\begin{array}{l}\text { Patient } \\
\text { safety }\end{array}$ & $\begin{array}{l}\text { Stuff } \\
\text { safety }\end{array}$ \\
\hline Missing sample and/or test request & $\begin{array}{l}\text { Computerized physician order entry and query-host } \\
\text { communication }\end{array}$ & $\sqrt{ }$ & - & $\sqrt{ }$ & - \\
\hline Wrong/missing identification & Positive patient identification & $\sqrt{ }$ & $\sqrt{ }$ & $\sqrt{ }$ & - \\
\hline In vitro hemolysis & $\begin{array}{l}\text { Usage of an adaptor, automated venous sampling system, } \\
\text { and real-time digital imager }\end{array}$ & $\sqrt{ }$ & $\sqrt{ }$ & $\sqrt{ }$ & - \\
\hline Wrong container & The tube labeler and preparer & $\sqrt{ }$ & $\sqrt{ }$ & $\sqrt{ }$ & - \\
\hline Insufficient sample & $\begin{array}{l}\text { Usage of an adaptor and the containers with a } \\
\text { pre-determined vacuum volume }\end{array}$ & $\sqrt{ }$ & $\sqrt{ }$ & $\sqrt{ }$ & - \\
\hline $\begin{array}{l}\text { Inappropriate blood to anticoagulant } \\
\text { ratio }\end{array}$ & $\begin{array}{l}\text { Usage of an adaptor and the containers with a } \\
\text { pre-determined vacuum volume }\end{array}$ & $\sqrt{ }$ & $\sqrt{ }$ & $\sqrt{ }$ & - \\
\hline $\begin{array}{l}\text { Inappropriate transport and storage } \\
\text { conditions }\end{array}$ & PTS & $\sqrt{ }$ & $\sqrt{ }$ & $\sqrt{ }$ & $\sqrt{ }$ \\
\hline $\begin{array}{l}\text { Inadequate label alignment on the } \\
\text { specimen tubes }\end{array}$ & The tube labeler and preparer & $\sqrt{ }$ & $\sqrt{ }$ & $\sqrt{ }$ & $\sqrt{ }$ \\
\hline $\begin{array}{l}\text { The low-resolution print quality of the } \\
\text { bar-code label }\end{array}$ & The tube labeler and preparer & $\sqrt{ }$ & $\sqrt{ }$ & $\sqrt{ }$ & $\sqrt{ }$ \\
\hline \multicolumn{6}{|l|}{$\begin{array}{l}\text { Intra-laboratory error types or } \\
\text { inconveniences }\end{array}$} \\
\hline \multicolumn{6}{|l|}{ Preanalytical } \\
\hline $\begin{array}{l}\text { Inappropriate centrifugation } \\
\text { conditions }\end{array}$ & Automated centrifugation & $\sqrt{ }$ & $\sqrt{ }$ & $\sqrt{ }$ & $\sqrt{ }$ \\
\hline $\begin{array}{l}\text { Errors from different manual } \\
\text { processes }\end{array}$ & $\begin{array}{l}\text { Decapper, aliquoter, bar-code labeler, and recapper } \\
\text { module in pre-analytical automation systems }\end{array}$ & $\sqrt{ }$ & - & $\sqrt{ }$ & $\sqrt{ }$ \\
\hline $\begin{array}{l}\text { Delay in sample processing steps in } \\
\text { laboratory }\end{array}$ & Primary tube usage & $\sqrt{ }$ & - & - & $\sqrt{ }$ \\
\hline $\begin{array}{l}\text { Delayed submission and inefficient } \\
\text { specimen traceability }\end{array}$ & An automatic tube loader and sorter usage & $\sqrt{ }$ & $\sqrt{ }$ & $\sqrt{ }$ & $\sqrt{ }$ \\
\hline Inability to process a primary tube & Automated systems processing primary tube & $\sqrt{ }$ & - & - & $\sqrt{ }$ \\
\hline $\begin{array}{l}\text { Errors from inadequate sample } \\
\text { integrity }\end{array}$ & $\begin{array}{l}\text { Volume/clotting/bubble-controlling unit in pre-analytical } \\
\text { automation. }\end{array}$ & $\sqrt{ }$ & $\sqrt{ }$ & $\sqrt{ }$ & $\sqrt{ }$ \\
\hline \multicolumn{6}{|l|}{ Postanalytical } \\
\hline $\begin{array}{l}\text { Inappropriate storage condition in } \\
\text { the posttest phase }\end{array}$ & The automated specimen storage and retrieval system & $\sqrt{ }$ & $\sqrt{ }$ & $\sqrt{ }$ & $\sqrt{ }$ \\
\hline $\begin{array}{l}\text { Impairment in secondary sample } \\
\text { traceability }\end{array}$ & $\begin{array}{l}\text { Secondary tube sorter module of intra- laboratory } \\
\text { non-analytical automation }\end{array}$ & $\sqrt{ }$ & $\sqrt{ }$ & $\sqrt{ }$ & $\sqrt{ }$ \\
\hline Delayed test reporting & Automatic verification & $\sqrt{ }$ & $\sqrt{ }$ & $\sqrt{ }$ & \\
\hline $\begin{array}{l}\text { The deceleration in total laboratory } \\
\text { automation }\end{array}$ & Process-controlling software & $\sqrt{ }$ & $\sqrt{ }$ & $\sqrt{ }$ & $\sqrt{ }$ \\
\hline
\end{tabular}

patient safety. Adaptor usage and using the containers with a pre-determined vacuum volume, which is possible, hampers insufficient sample. During sampling, an adaptor is used for sampling, and complete filling of the tube vacuum lasted, precluding inappropriate blood to anticoagulant ratio. Inappropriate transport and storage conditions may be put an end to by PTS, which is closely associated with improved TAT and high-quality testing. The tube labeler and preparer restrains poor label alignment on the specimen container, especially in outpatients, reducing TAT. Automated centrifugation prevents inappropriate centrifugation conditions, shortening TAT, and providing staff safety.
The automated specimen storage and retrieval system anticipates the inappropriate storage condition of the samples in the posttest phase, meaning sample safety and improved TAT. An automatic tube loader and sorter usage can avert the delay in sorting and submission of the specimens, reducing TAT, achieving staff safety, and providing efficient specimen traceability. A delay in sample processing steps in the laboratory may be restricted by primary tube usage, which shortens TAT and provides direct storage within the automated cabinet. Volume/clotting/bubble sensors contained in pre-analytical automation systems counteract the errors originated from inadequate sample integrity, improving the 
test results, and TAT. Some errors coming from different manual processes are prevented by the module of decapper, aliquoter, bar-code labeler, and recapper contained in pre-analytical automation systems, which is associated with staff safety and TAT reduction. Impairment in secondary sample traceability is avoided by secondary tube sorter module of intra-laboratory non-analytical automation, relating to patient safety and reduced TAT. Delayed test reporting is counteracted by automatic verification, meaning improved TAT. Process-controlling software managing the system precludes the deceleration in total laboratory automation, resulting in high-quality test reporting.

Table 5 summarizes extra-analytical errors, improvements by automation, and improved parameters, including TAT, test result quality, patient safety, and staff safety. These four parameters are principal factors determining the performance of a clinical laboratory, proving the importance of non-analytical automation for high-quality laboratory performance.

The management and standardization of non-analytical laboratory processes, especially of pre-analytical ones, have been primarily achieved thanks recently to automation, either by stand-alone devices or those integrated with analytical automation, as well as by informatics and computer sciences. Non-analytical automated systems should be implemented in the laboratory processes. For this purpose, a risk management strategy should first be developed for the systematic analysis of the present laboratory's workflow and bottlenecks. Then, the measurements of improvements can be executed by the pathways of QIs and outcome measures. Non-analytical automation within and outside the clinical laboratory will standardize as possible as the processes in that phase. The standardization of a process within TTP will necessarily make it possible to control, to trace, to measure, to evaluate, and to improve it, providing the integration of TTP with maximal effectiveness, and all factors improved result in a high-quality test result reporting.

\section{Conclusion}

The considerations mentioned above suggest that the clinical laboratory automation and computer sciences have rendered non-analytical laboratory processes mostly manageable and standardized the automation technologies have added a serious impact on the proficiency of laboratory medicine. The laboratory processes need the implementation of a non-analytical automated system. A risk management strategy, for this reason, should first be developed for the systematic analysis of the present laboratory's workflow and bottlenecks. Then, the application of QIs for different steps allows measuring the improvements in TTP. Several manual processes have been automated by instrumentations, resulting in improvements in standardization, organization, efficiency, and quality of TTP. Consequently, non-analytical automation, within and outside the clinical laboratory, will permanently reduce the effect of the error sources in TTP and enhance the test result quality. More studies, or QI-oriented measurements, should be executed on the improvements in extra-analytical steps by non-analytical automation improvements, achieving accurate and faster test result reporting.

Research funding: None declared.

Author contributions: All authors have accepted responsibility for the entire content of this manuscript and approved its submission.

Competing interests: Authors state no conflict of interest.

\section{References}

1. Bakan E, Ozturk N, Baygutalp NK. Automation in the clinical laboratory: integration of several analytical and intra-laboratory pre- and post-analytical systems. Turk J Biochem 2017;42: 1-13.

2. Boyd JC, Felder RA. Pre-analytical automation in the clinical laboratory. In: Ward-Cook KM, Lehmann CA, Schoeff LE, Williams RH, editors. Clinical diagnostic technology: the total testing process, the pre-analytical phase. Washington DC: AACC Press; 2002, vol 1.

3. Rifai, N. Tietz textbook of clinical chemistry and molecular diagnostics - E-book, Kindle locations: 40565, 40595, 40607, 40623, 40630, 40667, 40684, 40688, and 40708, Kindle edition. In: Rifai N, Horvath AR, Wittwer CT, editors, Tietz, textbook of clinical chemistry and molecular diagnostics; 2018:6 p.

4. Boyd J. Robotic laboratory automation. Science 2002;295: 517-18.

5. Hawkins R. Managing the pre- and post-analytical phases of the total testing process. Ann Lab Med 2012;32:5-16.

6. Durner J. Clinical chemistry: challenges for analytical chemistry and the nanosciences from medicine. Angew Chem Int Ed Engl 2010;49:1026-51.

7. Lippi G, Chance JJ, Church S, Dazzi P, Fontana R, Giavarina D, et al. Pre-analytical quality improvement: from dream to reality. Clin Chem Lab Med 2011;49:1113-26.

8. Automated venous sampling system. Available from: https:// vasculogic.com/venouspro.html [Accessed Mar 2020].

9. Real-time digital imager. Available from: https://www.accuvein. com/products/catalog/av500-vein-viewing-system/ [Accessed Mar 2020].

10. Clinical and Laboratory Standards Institute. Specimen labels: content and location, fonts, and label orientation. CLSI Approved Standard AUT012-A. Wayne, PA: Clinical and Laboratory Standards Institute; 2011. 
11. Clinical and Laboratory Standards Institute. Laboratory automation: bar codes for specimen container identification. CLSI Approved Standard AUT002-A2. Wayne, PA: Clinical and Laboratory Standards Institute; 2005.

12. Snyder ML, Carter A, Jenkins K, Fantz CR. Patient misidentifications caused by errors in standard bar code technology. Clin Chem 2010;56:1554-60.

13. Hawker CD. Bar codes may have poorer error rates than commonly believed. Clin Chem 2010;56:1513-14.

14. Tube labeler and preparer. Available from: http://www. technomedica.co.jp [Accessed Mar 2020].

15. Tube labeler and preparer. Available from: http:// gelecekmuhendislik.com/futurelab-labeler/ [Accessed Mar 2020].

16. Pneumatic-tube-systems. Available from: https://aerocom.de/ en/pneumatic-tube-systems-products/ [Accessed Mar 2020].

17. Pneumatic-tube-systems. Available from: https://www.air-log. com/ [Accessed Mar 2020].

18. Pneumatic-tube-system products. Available from: https:// aerocom.de/en/pneumatic-tube-systems-products/ [Accessed Mar 2020].

19. Sasaki M, Kageoka T, Ogura K, Kataoka H, Ueta T, Sugihara S. Total laboratory automation in Japan: past, present, and the future. Clin Chim Acta 1998;278:217-27.

20. Tube loader and sorter. Available from: http:// gelecekmuhendislik.com/futurelab-sorter/ [Accessed Mar 2020].

21. Plasma tube with physical separator. Available from: https:// www.bd.com/europe/ [Accessed Mar 2020].

22. Clinical and Laboratory Standards Institute. Laboratory automation: communications with automated clinical laboratory systems, instruments, devices, and information systems. CLSI Approved Standard AUTO03-A2. Wayne, PA: Clinical and Laboratory Standards Institute; 2009.

23. Clinical and Laboratory Standards Institute. Auto verification of clinical laboratory test results. CLSI Approved Guideline AUTO10-A. Wayne, PA: Clinical and Laboratory Standards Institute; 2006.

24. Wiwanitkit V. Types and frequency of pre-analytical mistakes in the first Thai ISO 9002: 1994 certified clinical laboratory, 6-month monitoring. BMC Clin Pathol 2001;1:5.

25. Guder W. Preanalytical factors and their influence on analytical quality specifications. Scand J Clin Lab Invest 1999;59:545-9.

26. Plebani M. Errors in clinical laboratories or errors in laboratory medicine? Clin Chem Lab Med 2006;44:750-9.

27. Streitberg GS, Bwititi PT, Angel L, Sikaris KA. Automation and expert systems in core clinical chemistry. J Assoc Lab Autom 2009;14:94-105.

28. Boyd JC, Hawker CD. Automation in the clinical laboratory. In: Burtis CA, Ashwood ER, Bruns DE, editors. Tietz textbook of clinical chemistry and molecular diagnostics. Elsevier: St Louis, Missouri; 2012:469-84 pp.

29. Sarkozi L, Simson E, Ramanathan L. The effects of total laboratory automation on the management of a clinical chemistry laboratory. Retrospective analysis of 36 years. Clin Chim Acta 2003;329:89-94.

30. Hawker CD, Garr SB, Hamilton LT, Penrose JR, Ashwood ER, Weiss RL. Automated transport and sorting system in a large reference laboratory: part 1. Evaluation of needs and alternatives and development of a plan. Clin Chem 2002;48:1751-60.

31. Melanson SE, Lindeman NI, Jarolim P. Selecting automation for the clinical chemistry laboratory. Arch Pathol Lab Med 2007;131: 1063-9. https://doi.org/10.1043/1543-2165(2007)131[1063: SAFTCC]2.0.CO;2.

32. Hawker CD, Roberts WL, Garr SB, Hamilton LT, Penrose JR, Ashwood ER, et al. Automated transport and sorting system in a large reference laboratory. Part 2: implementation of the system and performance measures over three years. Clin Chem 2002;48: 1761-7.

33. Da Rin G. Pre-analytical workstations: a tool for reducing laboratory errors. Clin Chim Acta 2009;404:68-74.

34. Wallin O, Soderberg J, Van Guelpen B, Stenlund H, Grankvist K, Brulin C. Preanalytical venous blood sampling practices demand improvement: a survey of test-request management, test-tube labeling, and information search procedures. Clin Chim Acta 2008;391:91-7.

35. Soderberg J, Brulin C, Grankvist K, Wallin O. Pre-analytical errors in primary healthcare: a questionnaire study of information search procedures, test request management, and test tube labeling. Clin Chem Lab Med 2009;47:195-201.

36. Kemp GM, Bird CE, Barth JH. Short-term interventions onwards fail to reduce pre-analytical errors: results of two prospective controlled trials. Ann Clin Biochem 2012;49:166-9.

37. Valestein P. Laboratory turnaround time. Am J Clin Pathol 1996; 105:676-88.

38. UNI EN ISO 15189. Medical laboratories: requirements for quality and competence. Geneva (Switzerland): International Organization for Standardization; 2013.

39. Plebani M. Quality indicators to detect pre-analytical errors in laboratory testing. Clin Biochem Rev 2012;33:85-8.

40. Wagar EA, Tamashiro L, Yasin B, Hilborne L, Bruckner DA. Patient safety in the clinical laboratory: a longitudinal analysis of specimen identification errors. Arch Pathol Lab Med 2006;130 1662-8. https://doi.org/10.1043/1543-2165(2006)130[1662: PSITCL]2.0.CO;2.

41. Lippi G, Blanckaert N, Bonini P, Green S, Kitchen S, Palicka V, et al. Causes, consequences, detection, and prevention of identification errors in laboratory diagnostics. Clin Chem Lab Med 2009;47:143-53.

42. Plebani M, Sciacovelli L, Aita A, Padoan A, Chiozza ML. Quality Indicators to detect pre-analytical errors in laboratory testing. Clin Chim Acta 2014;432:44-8.

43. Plebani M, Sciacovelli L, Marinova M, Marcuccitti J, Chiozza ML. Quality indicators in laboratory medicine: a fundamental tool for quality and patient safety. Clin Biochem 2013;46: 1170-4.

44. Sciacovelli L, Plebani M. The IFCC Working Group on laboratory errors and patient safety. Clin Chim Acta 2009;404:79-85.

45. Plebani M, Astion ML, Barth JH, Chen W, de Oliveira Galoro CA, Escuer MI, et al. Harmonization of quality indicators in laboratory medicine. A preliminary consensus. Clin Chem Lab Med 2014;52: 951-8.

46. Plebani M, Sciacovelli L, Aita A. Quality indicators for the total testing process. Biol Sci Clin Lab Med 2017;37:187-205. 